\title{
Preparation and Characterization of Aligned Iron Oxide Carbon Nanotube Thin Film
}

\begin{abstract}
H. Hekmatara, M. Seifi* and S.M. Rozati
Department of Physics, Faculty of Science, University of Guilan, Rasht, Iran

(Received January 30, 2013; revised version November 9, 2013; in final form November 18, 2013)

Thin films of acid-functionalized multiwall carbon nanotubes (O-MWCNT) with different concentrations and coated O-MWCNT with $\mathrm{Fe}_{3} \mathrm{O}_{4}$ nanoparticles (MWCNT $/ \mathrm{Fe}_{3} \mathrm{O}_{4}$ ) were prepared on glass substrate at $300^{\circ} \mathrm{C}$ by spray pyrolysis technique. In order to study the effect of nanotubes alignment on the physical properties of carbon nanotube films, thin film of iron oxide nanoparticles coated carbon nanotubes was deposited under magnetic field of $0.4 \mathrm{~T}$. All samples were characterized using UV-Vis spectroscopy, X-ray diffraction, scanning electron microscopy, and the Hall effect experiment. Results show that the electrical conductivity and optical transmittance of carbon nanotubes thin films depend on the concentration of carbon nanotubes and their arrangement at the films. Aligning carbon nanotubes in thin films leads to an obvious improvement in electrical and optical properties of thin films.
\end{abstract}

DOI: 10.12693/APhysPolA.125.77

PACS 73.63.Fg, 73.61.-r

\section{Introduction}

Anisotropic properties of graphite along $a$-plane and $c$-plane directions lead to anisotropy in physical properties of carbon nanotubes $[1,2]$. Because of this strong anisotropy of CNT properties, it is always necessary to consider the orientation of nanotube's axis when a CNT component is considered for both scientific studies and practical applications. Anisotropic nature of individual CNTs is best preserved and explicitly conceived when all the CNTs are aligned in the same direction as an array and making it possible to control the morphology of each of them. So it is critical in most applications to know and control how a CNT is oriented either as a stand-alone individual or in a group of many CNTs bundles. It is obviously more challenging and more desirable to obtain a CNT ensemble with all its members having a common orientation. There are some of technologies available to align a single or an array of CNTs along a predetermined orientation. The techniques rely on different mechanisms and therefore are applicable for different situations. These techniques are categorized into two groups pertaining to when the alignment is achieved: (a) in situ techniques where alignment is achieved during the CNT growth process, (b) ex situ techniques where CNTs are originally grown in random orientations and alignment is achieved afterwards such as during the device integration process [3-5].

In the ex situ methods nanotubes are usually exposed to an external electric and magnetic fields, these methods are preferred because of their independence from growth limited conditions and easy controllable [6,9]. In the recent researches, vertically aligning nanotubes on the sub-

*corresponding author; e-mail: m_seifi2000@yahoo.com strate have only been performed via in situ techniques $[10,11]$. This arrangement avoids tube-tube contacts, which can limit charge transport through film [12, 13]. In this paper, thin films of: acid functionalized multiwall carbon nanotubes (O-MWCNTs), coated O-MWCNTs with $\mathrm{Fe}_{3} \mathrm{O}_{4}$ nanoparticles (MWCNT $/ \mathrm{Fe}_{3} \mathrm{O}_{4}$ ), and also aligned MWCNT $/ \mathrm{Fe}_{3} \mathrm{O}_{4}$ under magnetic field were produced. The observations show that the distribution of deposited O-MWCNTs on the glass substrate was uniform. But in MWCNT $/ \mathrm{Fe}_{3} \mathrm{O}_{4}$ film, uniform distribution of nanotubes is broken. It has also been obtained that the electrical conductivity of $\mathrm{MWCNT} / \mathrm{Fe}_{3} \mathrm{O}_{4}$ film comparing to O-MWCNT film is decreased. In aligned $\mathrm{Fe}_{3} \mathrm{O}_{4}$ coated MWCNT film, the electrical conductivity was increased. Transmission spectra show that, in aligned MWCNT $/ \mathrm{Fe}_{3} \mathrm{O}_{4}$ film, the transparency was increased over $20 \%$ respect to the random oriented MWCNT/ $\mathrm{Fe}_{3} \mathrm{O}_{4}$ film.

\section{Experimental}

Thin films of O-MWCNTs were deposited on glass substrate at $300^{\circ} \mathrm{C}$ using spray pyrolysis method. The starting suspension for spray process was prepared by dispersing $0.005 \mathrm{~g}$ acid functionalized MWCNT in $20 \mathrm{ml}$ of ethanol/water $(3 / 1)$ solution. The solution was sonicated for about 90 min until a homogeneous and stable suspension was achieved. Then the film was deposited in air at a flow rate of $20 \mathrm{~L} / \mathrm{m}$ and a distance from substrate to nozzle $25 \mathrm{~cm}$. In order to study the effect of various treatments on the properties of O-MWCNT film, four samples were studied: first sample contains $0.005 \mathrm{~g}$ O-MWCNT, sample 2 contains $0.01 \mathrm{~g}$ acid functionalized MWCNT. Iron oxide nanoparticles coated multiwall carbon nanotubes (MWCNT/ $/ \mathrm{Fe}_{3} \mathrm{O}_{4}$ ) were produced as follows: $96 \mathrm{mg}$ O-MWCNT was mixed with $81 \mathrm{mg}$ $\mathrm{FeCl}_{3} \cdot 6 \mathrm{H}_{2} \mathrm{O}$ and $120 \mathrm{mg} \mathrm{FeCl} 2 \cdot 4 \mathrm{H}_{2} \mathrm{O}$ in deionized water under argon atmosphere. The mixture was stirred 
for 30 min; subsequently dilute solution of ammonia was added dropwise to the mixture until the $\mathrm{pH}$ raised to 12 . The synthesis process was followed by heating the mixture to $60^{\circ} \mathrm{C}$. The reaction was completed after $2 \mathrm{~h}$. The resulting composite was then washed and dried at $100{ }^{\circ} \mathrm{C}$ in air [14]. Sample 3 contains $0.005 \mathrm{~g} \mathrm{MWCNT/}$ $\mathrm{Fe}_{3} \mathrm{O}_{4}$. The process of making aligned MWCNT $/ \mathrm{Fe}_{3} \mathrm{O}_{4}$ film (sample 4) was the same as sample 3 , but in order to align nanotubes at the film, a magnetic field of $0.4 \mathrm{~T}$ was used. Optical transmittance spectra of the films were measured using a UV-Vis double beam spectrophotometer by Cary100 with air as reference. The Hall effect setup supplied by Scientific Equipments, Phys. Tech. RH 2010 was used for electrical measurements by using Van der Pauw configuration at room temperature.

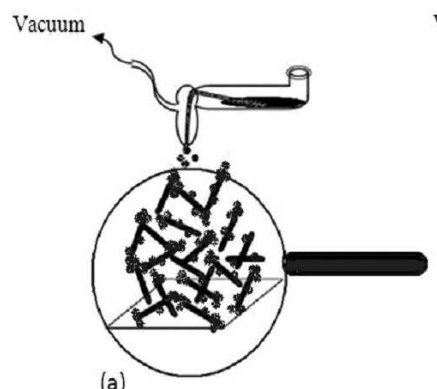

(a)

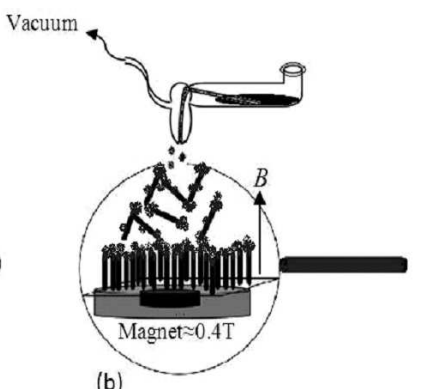

(b)
Fig. 1. Deposition processes of films that contain (a) MWCNT $/ \mathrm{Fe}_{3} \mathrm{O}_{4}$ and (b) MWCNT $/ \mathrm{Fe}_{3} \mathrm{O}_{4}$ in the presence of magnetic field.

X-ray diffraction (XRD) method was applied to determine the crystalline quality of prepared films by using $\mathrm{Cu} K_{\alpha}$ radiation Philips PW-1830 model. Scanning electron microscopy (SEM) was carried out by VEGA $\backslash \backslash$ TESCAN-LMU model for morphological analysis of the films. The thickness of films was calculated from the Swanepoel method by using standard formula [15]. The schematic image from deposition process of $\mathrm{MWCNT} / \mathrm{Fe}_{3} \mathrm{O}_{4}$ thin films is illustrated in Fig. 1.

\section{Result and discussion}

\subsection{TEM and SEM}

The produced thin films were characterized using SEM (VEGA $\backslash \backslash$ TESCAN-LMU model) and TEM (PHILIPS CM 10). With the present process condition we observed a large number of iron oxide nanoparticles in the sample. Figure 2 shows the TEM image of MWCNT coated with $\mathrm{Fe}_{3} \mathrm{O}_{4}$ nanoparticles. Here, the internal diameter of carbon nanotubes ranges from 10 to $20 \mathrm{~nm}$, and their average length is $30 \mu \mathrm{m}$. It is clear that the distribution of iron oxide nanoparticles is not uniform in the whole sample; the most of nanoparticles were placed at the open tips of carbon nanotubes in the form of clusters. It is attributed to the reactivity of nanotubes tips because of the highest density of functional groups. It is also observed that several number of small $\mathrm{Fe}_{3} \mathrm{O}_{4}$ nanoparticles attached to the CNT's surface.

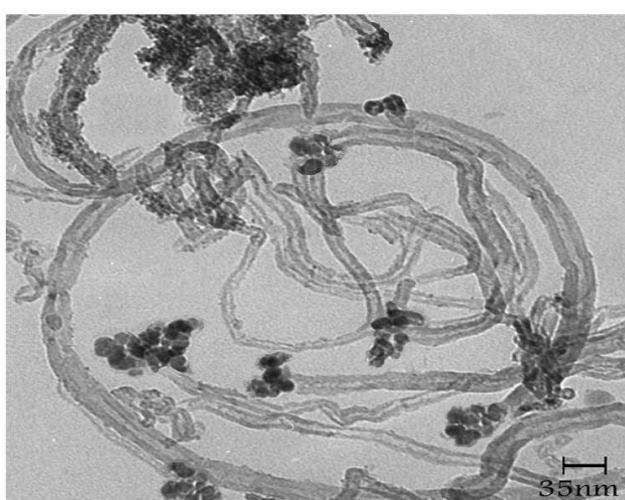

Fig. 2. TEM image of iron oxide coated multiwall carbon nanotubes (MWCNT/ $\mathrm{Fe}_{3} \mathrm{O}_{4}$ ).

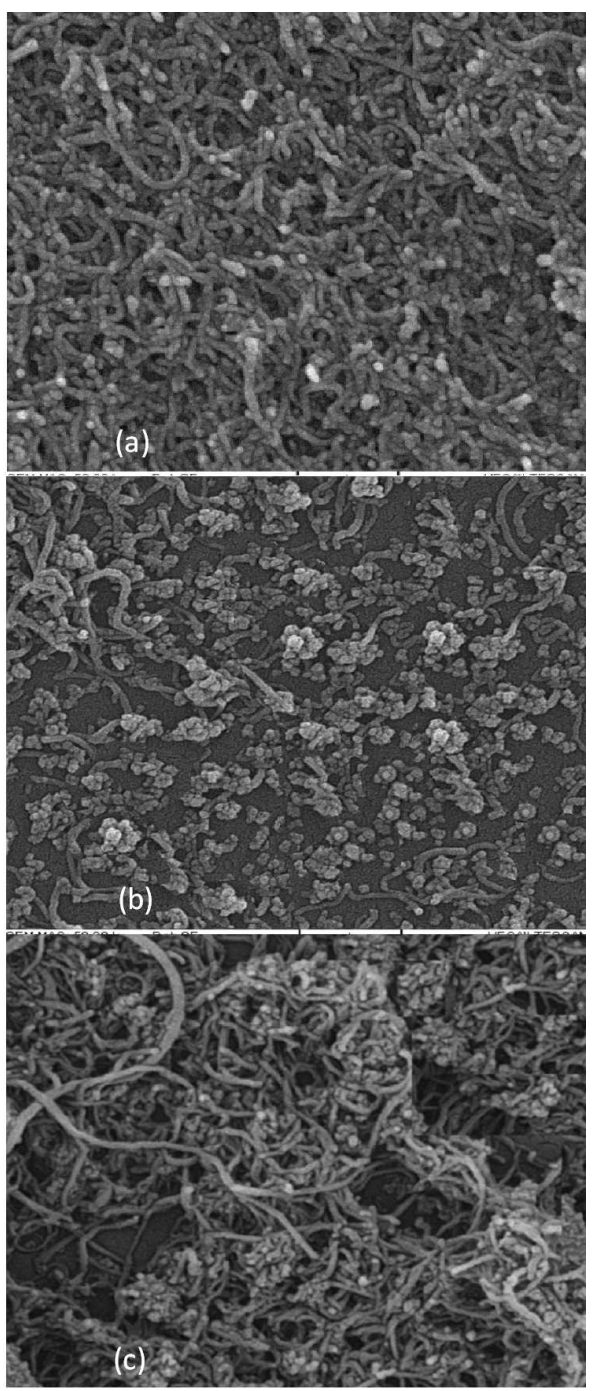

Fig. 3. SEM images of samples: (a) O-MWCNT, (b) $\mathrm{MWCNT} / \mathrm{Fe}_{3} \mathrm{O}_{4}$ films and (c) $\mathrm{MWCNT} / \mathrm{Fe}_{3} \mathrm{O}_{4}$ which were prepared in the presence of magnetic field. 
Figure 3 shows the SEM images of total samples. Figure 3a shows the film of O-MWCNTs. In this film it was observed that the nanotubes are uniformly arranged at the film. As observed in Fig. 3b, the uniform distribution of carbon nanotubes is broken. Figure $3 \mathrm{c}$ shows the image of carbon nanotubes coated with iron oxide film under magnetic field. The nanotubes are aligned in the direction of magnetic field (vertically to the substrate). This image shows the top view of the film, only the tips of nanotubes that were coated with iron oxide nanoparticles are observable. It will also be noted that the separation of nanotubes in the film was increased by applying magnetic field which is in good agreement with the previous reports [16].

\subsection{XRD pattern}

The XRD pattern of the films is shown in Fig. 4. The crystalline surface of nanotubes in sample of O-MWCNT thin film are placed at $2 \theta=26^{\circ}$ and $32^{\circ}$ which belongs to [002] and [100] surfaces (Fig. 4a). The peaks of thin films are a bit weaker than peaks of bulk nanotubes [17]. The XRD pattern of sample 2, Fig. 4b, shows a similar profile with higher intensity due to enhancement of nanotubes concentration in the film. Figure $4 \mathrm{c}$ shows the pattern of sample 3, containing coated nanotubes with iron oxide. The large numbers of peaks corresponding to MWCNT and iron oxide nanoparticles are also observed. The MWCNTs peaks are shifted to higher angles. The XRD pattern of aligned nanotubes (Fig. 4d) shows that the peaks of iron oxide nanoparticles are shifted to the higher angles with respect to $\mathrm{MWCNT} / \mathrm{Fe}_{3} \mathrm{O}_{4}$ without any changes in MWCNT peaks.

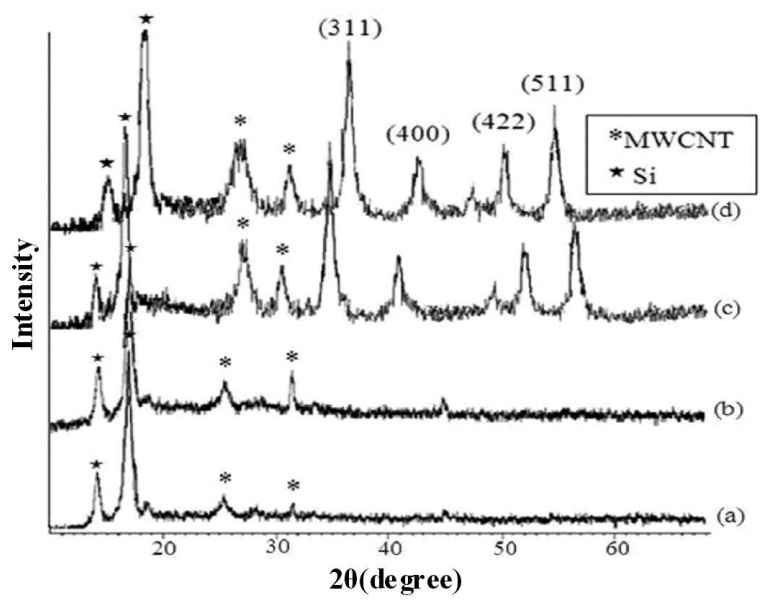

Fig. 4. XRD patterns of (a) O-MWCNT (0.005 g), (b) $\mathrm{O}-\mathrm{MWCNT}(0.01 \mathrm{~g}),(c) \mathrm{MWCNT} / \mathrm{Fe}_{3} \mathrm{O}_{4}$ films, and $(d)$ $\mathrm{MWCNT} / \mathrm{Fe}_{3} \mathrm{O}_{4}$ film which were prepared in the presence of magnetic field.

\subsection{Optical properties}

Transmission spectra of all prepared samples in the $\mathrm{UV}-\mathrm{V}$ is region have been illustrated in Fig. 5. The spectrum of the films does not show any transparency in the ultraviolet region between 200 to $305 \mathrm{~nm}$. But in the range of visible wavelengths and near visible ranges (310-400 nm), all samples are highly transparent. Having this special property makes carbon nanotubes thin films as a good option for manufacturing sunscreen glasses and solar cells. We observed that the transparency of O-MWCNT depends on the nanotube concentration in the film. This figure shows that, by increasing the nanotube concentration, the transparency of the film decreased. It will be noted that coating carbon nanotubes with iron oxide nanoparticles leads to reduction in transparency of the film. It is assigned to the reflective nature of iron oxide nanoparticles in visible and near visible range. It has also been observed that the transparency increased by aligning carbon nanotubes in the film. As shown in the SEM image, applied magnetic field separated the nanotubes in the film and created lots of pores in the film which all are completely transparent.

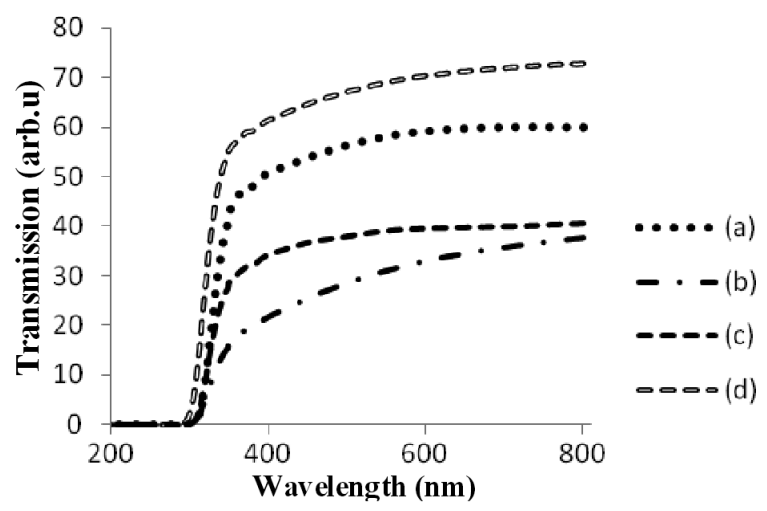

Fig. 5. Optical transmittance of (a) O-MWCNT (0.005 g), (b) O-MWCNT (0.01 g), (c) MWCNT $/ \mathrm{Fe}_{3} \mathrm{O}_{4}$ films and $(d)$ MWCNT $/ \mathrm{Fe}_{3} \mathrm{O}_{4}$ film which were prepared in the presence of magnetic field.

\subsection{Measuring the thicknesses of all thin films by Swanepoel's method}

The method suggested by Swanepoel was used to measure the thickness of all thin films. The upper and lower envelopes of the interference fringes were obtained from the transmission spectrum. Refractive index calculation was performed by the following equation:

$$
n=\sqrt{N+\sqrt{N^{2}-S^{2}}},
$$

where $S$ is the refractive index of the substrate and $N$ is

$$
N=2 s \frac{T_{\mathrm{M}}-T_{\mathrm{m}}}{T_{\mathrm{M}} T_{\mathrm{m}}}+\frac{s^{2}+1}{2} .
$$

$T_{\mathrm{M}}=$ value of transmission maximum at the wavelengths of the upper envelopes, $T_{\mathrm{m}}=$ value of transmission minimum at the wavelengths of the lower envelopes.

The maximum absolute accuracy of $T_{\mathrm{M}}$ and $T_{\mathrm{m}}$ is \pm 0.001 . Also, if $n_{1}$ and $n_{2}$ are the refractive indices at two adjacent maxima (or minima) at wavelengths $\lambda_{1}$ and $\lambda_{2}$, using the basic equation for interference fringes 
$2 n d=m \lambda$,

where $m$ is an integer for maxima and half integer for minima, the thickness $(d)$ is given by

$$
d=\frac{\lambda_{1} \lambda_{2}}{4\left(\lambda_{1} n_{2}-\lambda_{2} n_{1}\right)} \text {. }
$$

\subsection{Electrical properties}

The electrical properties, including sheet resistance, resistivity, carrier concentration and mobility of thin films were calculated using the Hall effect experiment. The obtained results show that the conductivity of O-MWCNT films depends on nanotubes concentration. The conductivity measurements also show that coating nanotubes with iron oxide nanoparticles decreased the conductivity of the films. Here the iron oxide nanoparticles played a role of scattering sites and increased the resistivity of the film (resistance $\approx 217 \Omega \mathrm{cm}$ ). This result is in agreement with electrical conductivity of the bulk sample of $\mathrm{MWCNT} / \mathrm{Fe}_{3} \mathrm{O}_{4}[18]$.

In order to measure the electrical conductivity of aligned $\mathrm{MWCNT} / \mathrm{Fe}_{3} \mathrm{O}_{4}$ thin film, along the nanotubes axis, $\mathrm{Fe}_{3} \mathrm{O}_{4}$ coated carbon nanotubes were stretched along the substrate plane using magnetic field and then placed between the poles of a magnet in the Hall effect setup. Optical microscopic image of this sample and schematic Hall effect setup for measuring the electrical conductivity of it are shown in Figs. 6 and 7, respectively. It could be observed that aligning nanotubes leads to increase in the electrical conductivity of the film. This can be related to the anisotropic electrical properties of nanotubes. The electrical conductivity of carbon nanotubes in nanotube axis direction is higher than radial direction.

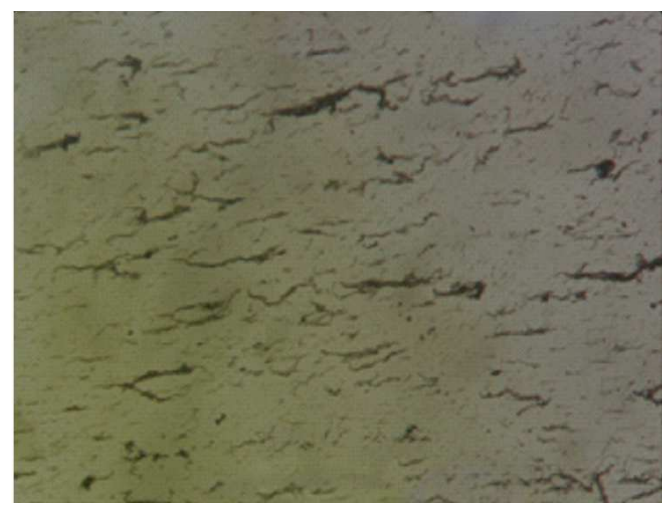

Fig. 6. Optical microscopic image of the sample prepared for measuring the electrical conductivity of MWCNT $/ \mathrm{Fe}_{3} \mathrm{O}_{4}$ along the nanotubes axis direction.

Table includes detailed information from concentrations, type and charge carrier mobility of total samples. As shown in this table, the number of carriers in coated nanotubes thin films increased. But the mobility of these carriers decreased. The Hall mobility diminution in coated samples might be related to induce scattering sites (doping nanoparticles on nanotubes sidewall). Moreover,

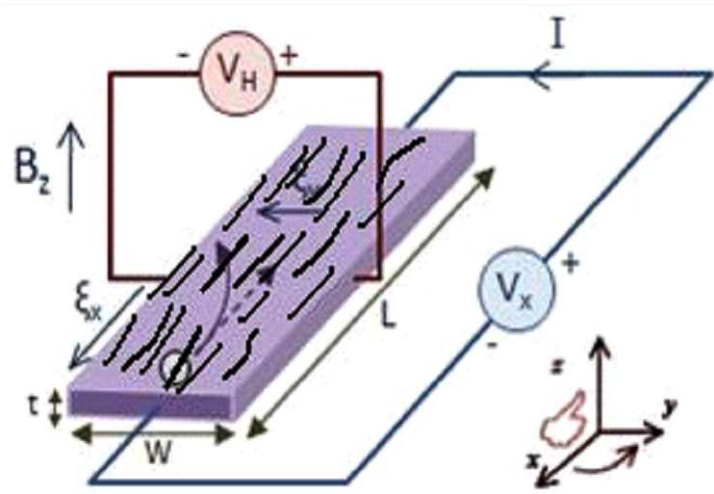

Fig. 7. Schematic image of Hall effect setup for measuring the electrical conductivity of $\mathrm{MWCNT} / \mathrm{Fe}_{3} \mathrm{O}_{4}$ along the nanotube axis direction.

Electrical properties of thin films.

TABLE

\begin{tabular}{c|c|c|c|c}
\hline \hline Sample & Thickness & $\rho[\Omega \mathrm{cm}]$ & $n\left[\mathrm{~cm}^{-3}\right]$ & $\mu\left[\mathrm{cm}^{2} /(\mathrm{V} \mathrm{s})\right]$ \\
\hline 1 & $5 \mu \mathrm{m}$ & 50 & $-9.59 \times 10^{+13}$ & $2.25 \times 10^{+3}$ \\
2 & $5 \mu \mathrm{m}$ & 30 & $-8.53 \times 10^{+13}$ & $1.3 \times 10^{+3}$ \\
3 & $5 \mu \mathrm{m}$ & 217 & $3.05 \times 10^{+16}$ & 2.73 \\
4 & $5 \mu \mathrm{m}$ & 70 & $4.47 \times 10^{+17}$ & $1.95 \times 10^{+1}$
\end{tabular}

coating nanotubes with iron oxide nanoparticles leads to change the charge carrier of MWCNT $/ \mathrm{Fe}_{3} \mathrm{O}_{4}$ from electron (minus) into hole (positive) carriers. Changing in carrier type was taken place as a result of doping nanotubes by $\mathrm{Fe}_{3} \mathrm{O}_{4}$ nanoparticles, that is known as $p$-type semiconductor [19].

\section{Conclusion}

Different samples of carbon nanotubes thin films which contained O-MWCNT with two concentrations and $\mathrm{MWCNT} / \mathrm{Fe}_{3} \mathrm{O}_{4}$ were prepared by spray pyrolysis process. SEM images of the films showed that the distribution of nanotubes in random oriented O-MWCNT films is uniform. By applying magnetic field, nanotubes are held in the field direction (vertically to the film substrate). Electrical and optical properties of thin films were investigated in this paper based on the Hall effect and transmission spectra in UV-Vis region. The obtained results showed that the electrical conductivity in the O-MWCNT films depends on the concentration of samples in the thin films.

Increment in carbon nanotubes concentration leads to increase in electrical conductivity of film. It was observed that the presence of iron oxide nanoparticles in the samples acts as a resistance, thus the conductivity of the films decreased. The results also proved that applying magnetic field during the deposition process caused increase in electrical conductivity of the films. Transmission spectra of the films do not show any transparency in the ultraviolet region. The spectra also show that the 
transparency of O-MWCNT films decreased by increasing the amount of O-MWCNT. It will be noted that carbon nanotubes alignment improved the transparency of the film.

\section{References}

[1] K. Hata, D.N. Futaba, K. Mizuno, T. Namai, M. Yumura, S. Iijima, Science 306, 1362 (2004).

[2] H.W. Zhu, C.L. Xu, D.H. Wu, B.Q. Wei, R. Vajtai, P.M. Ajayan, Science 296, 8842 (2002).

[3] E.S. Choi, J.S. Brooks, D.L. Eaton, M.S. AlHaik, M.Y. Hussaini, H. Garmestani, D.K. Dahmen, J. Appl. Phys. 94, 6034 (2003).

[4] A. Sharma, S. Kumar, B. Tripathi, M. Singh, Y.K. Vijay, Int. J. Hydrogen En. 34, 3977 (2009).

[5] Y. Ma, B. Wang, Y. Wu, Y. Huang, Y. Chen, Carbon 49, 4098 (2011).

[6] B.M. Quinn, S.G. Lemay, Adv. Mater. 18, 855 (2006).

[7] A. Naeemi, J.D. Meindl, IEEE Trans. Electron. Dev. 54, 26 (2007).

[8] H. Cho, K.H. Koo, P. Kapur, K.C. Saraswat, IEEE Electron. Dev. Lett. 29, 122 (2008).
[9] R. Krupke, F. Hennrich, H. von Lohneysen, M.M. Kappes, Science 301, 344 (2003).

[10] M.S. Fuhrer, J. Nygard, L. Shih, M. Forero, Y.G. Yoon, M.S.C. Mazzoni, H.J. Choi, J. Ihm, S.G. Louie, A. Zettl, P.L. McEuen, Science 288, 494 (2000).

[11] A.A. Odintsov, Phys. Rev. Lett. 85, 150 (2000).

[12] R. Krupke, S. Linden, M. Rapp, F. Hennrich, Adv. Mater. 18, 1468 (2006).

[13] M.D. Lima, M.J. de Andrade, C.P. Bergmann, S. Roth, J. Mater. Chem. 18, 776 (2008).

[14] L. Kong, X. Lub, W. Zhang, J. Solid State Chem. 181, 628 (2008).

[15] R. Swanepoel, J. Phys. E 16, 1214 (1983).

[16] Y. Lan, Y. Wang, Z.F. Ren, Advances in Physics, 60, 553 (2011).

[17] Q. Zhang, M. Zhu, Q. Zhang, Y. Li, H. Wang, Compos. Sci. Technol. 69,633 (2009).

[18] Li. Hsu , Y. Li, C. Hsiao, Nanosc. Res. Lett. 3, 330 (2008).

[19] V. Lisauskas, K. Suziene, R. Butkute, S. Tamulevicius, M. Andrulevicius, B. Vengalis, Acta Phys. Pol. A 113, 1055 (2008). 